\title{
Improved Direct Measurement of Leptonic Coupling Asymmetries with Polarized Z Bosons
}

Koya Abe,${ }^{24}$ Kenji Abe, ${ }^{15}$ T. Abe,${ }^{21}$ I. Adam, ${ }^{21}$ H. Akimoto, ${ }^{21}$ D. Aston, ${ }^{21}$ K. G. Baird, ${ }^{11}$ C. Baltay, ${ }^{30}$ H. R. Band, ${ }^{29}$ T. L. Barklow, ${ }^{21}$ J. M. Bauer, ${ }^{12}$ G. Bellodi, ${ }^{17}$ R. Berger,${ }^{21}$ G. Blaylock, ${ }^{11}$ J. R. Bogart, ${ }^{21}$ G. R. Bower, ${ }^{21}$ J. E. Brau, ${ }^{16}$ M. Breidenbach, ${ }^{21}$ W. M. Bugg, ${ }^{23}$ D. Burke, ${ }^{21}$ T. H. Burnett, ${ }^{28}$ P. N. Burrows, ${ }^{17}$ A. Calcaterra,${ }^{8}$ R. Cassell, ${ }^{21}$ A. Chou, ${ }^{21}$ H. O. Cohn, ${ }^{23}$ J. A. Coller, ${ }^{4}$ M. R. Convery, ${ }^{21}$ V. Cook,${ }^{28}$ R. F. Cowan, ${ }^{13}$ G. Crawford, ${ }^{21}$ C. J. S. Damerell, ${ }^{19}$ M. Daoudi, ${ }^{21}$ N. de Groot,${ }^{2}$ R. de Sangro,${ }^{8}$ D. N. Dong, ${ }^{21}$ M. Doser, ${ }^{21}$ R. Dubois,${ }^{21}$ I. Erofeeva,${ }^{14}$ V. Eschenburg, ${ }^{12}$ E. Etzion, ${ }^{29}$ S. Fahey, ${ }^{5}$ D. Falciai, ${ }^{8}$ J. P. Fernandez,${ }^{26}$ K. Flood,,${ }^{11}$ R. Frey, ${ }^{16}$ E. L. Hart,${ }^{23}$ K. Hasuko, ${ }^{24}$ S. S. Hertzbach, ${ }^{11}$ M. E. Huffer, ${ }^{21}$ X. Huynh, ${ }^{21}$ M. Iwasaki, ${ }^{16}$ D. J. Jackson, ${ }^{19}$ P. Jacques, ${ }^{20}$ J. A. Jaros, ${ }^{21}$ Z. Y. Jiang, ${ }^{21}$ A. S. Johnson, ${ }^{21}$ J. R. Johnson, ${ }^{29}$ R. Kajikawa, ${ }^{15}$ M. Kalelkar, ${ }^{20}$ H. J. Kang, ${ }^{20}$ R. R. Kofler, ${ }^{11}$ R. S. Kroeger, ${ }^{12}$ M. Langston, ${ }^{16}$ D. W. G. Leith, ${ }^{21}$ V. Lia, ${ }^{13}$ C. Lin, ${ }^{11}$ G. Mancinelli, ${ }^{20}$ S. Manly, ${ }^{30}$ G. Mantovani,,${ }^{18}$ T. W. Markiewicz, ${ }^{21}$ T. Maruyama, ${ }^{21}$ A. K. McKemey, ${ }^{3}$ R. Messner, ${ }^{21}$ K. C. Moffeit, ${ }^{21}$ T. B. Moore, ${ }^{30}$ M. Morii, ${ }^{21}$ D. Muller, ${ }^{21}$ V. Murzin, ${ }^{14}$ S. Narita, ${ }^{24}$ U. Nauenberg, ${ }^{5}$ H. Neal,,${ }^{30}$ G. Nesom, ${ }^{17}$ N. Oishi, ${ }^{15}$ D. Onoprienko, ${ }^{23}$ L. S. Osborne, ${ }^{13}$ R. S. Panvini, ${ }^{27}$ C. H. Park, ${ }^{22}$ I. Peruzzi, ${ }^{8}$ M. Piccolo, ${ }^{8}$ L. Piemontese, ${ }^{7}$ R. J. Plano, ${ }^{20}$ R. Prepost,${ }^{29}$ C. Y. Prescott, ${ }^{21}$ B. N. Ratcliff, ${ }^{21}$ J. Reidy, ${ }^{12}$ P. L. Reinertsen, ${ }^{26}$ L. S. Rochester, ${ }^{21}$ P. C. Rowson, ${ }^{21}$ J. J. Russell, ${ }^{21}$ O. H. Saxton, ${ }^{21}$ T. Schalk, ${ }^{26}$ B. A. Schumm, ${ }^{26}$ J. Schwiening, ${ }^{21}$ V. V. Serbo, ${ }^{21}$ G. Shapiro, ${ }^{10}$ N. B. Sinev, ${ }^{16}$ J. A. Snyder, ${ }^{30}$ H. Staengle, ${ }^{6}$ A. Stahl, ${ }^{21}$ P. Stamer, ${ }^{20}$ H. Steiner, ${ }^{10}$ D. Su, ${ }^{21}$ F. Suekane, ${ }^{24}$ A. Sugiyama, ${ }^{15}$ S. Suzuki, ${ }^{15}$ M. Swartz, ${ }^{9}$ F. E. Taylor, ${ }^{13}$ J. Thom,${ }^{21}$ E. Torrence, ${ }^{13}$ T. Usher ${ }^{21}$ J. Va' vra, ${ }^{21}$ R. Verdier, ${ }^{13}$ D. L. Wagner, ${ }^{5}$ A. P. Waite, ${ }^{21}$ S. Walston, ${ }^{16}$ A. W. Weidemann ${ }^{23}$ E. R. Weiss, ${ }^{28}$ J. S. Whitaker, ${ }^{4}$ S. H. Williams, ${ }^{21}$ S. Willocq,${ }^{11}$ R. J. Wilson, ${ }^{6}$ W. J. Wisniewski, ${ }^{21}$ J. L. Wittlin, ${ }^{11}$ M. Woods, ${ }^{21}$ T. R. Wright, ${ }^{29}$ R. K. Yamamoto, ${ }^{13}$ J. Yashima, ${ }^{24}$ S. J. Yellin, ${ }^{25}$ C. C. Young, ${ }^{21}$ and H. Yuta ${ }^{1}$

(The SLD Collaboration)

\author{
${ }^{1}$ Aomori University, Aomori, 030 Japan \\ ${ }^{2}$ University of Bristol, Bristol, United Kingdom \\ ${ }^{3}$ Brunel University, Uxbridge, Middlesex UB8 3PH United Kingdom \\ ${ }^{4}$ Boston University, Boston, Massachusetts 02215 \\ ${ }^{5}$ University of Colorado, Boulder, Colorado 80309 \\ ${ }^{6}$ Colorado State University, Ft. Collins, Colorado 80523 \\ ${ }^{7}$ INFN Sezione di Ferrara and Universita di Ferrara, I-44100 Ferrara, Italy \\ ${ }^{8}$ INFN Laboratori Nazionali di Frascati, I-00044 Frascati, Italy \\ ${ }^{9}$ Johns Hopkins University, Baltimore, Maryland 21218-2686 \\ ${ }^{10}$ Lawrence Berkeley Laboratory, University of California, Berkeley, California 94720 \\ ${ }^{11}$ University of Massachusetts, Amherst, Massachusetts 01003 \\ ${ }^{12}$ University of Mississippi, University, Mississippi 38677 \\ ${ }^{13}$ Massachusetts Institute of Technology, Cambridge, Massachusetts 02139 \\ ${ }^{14}$ Institute of Nuclear Physics, Moscow State University, 119899, Moscow, Russia \\ ${ }^{15}$ Nagoya University, Chikusa-ku, Nagoya, 464 Japan \\ ${ }^{16}$ University of Oregon, Eugene, Oregon 97403 \\ ${ }^{17}$ Oxford University, Oxford OX1 3RH, United Kingdom \\ ${ }^{18}$ INFN Sezione di Perugia and Universita di Perugia, I-06100 Perugia, Italy \\ ${ }^{19}$ Rutherford Appleton Laboratory, Chilton, Didcot, Oxon OX11 OQX United Kingdom \\ ${ }^{20}$ Rutgers University, Piscataway, New Jersey 08855 \\ ${ }^{21}$ Stanford Linear Accelerator Center, Stanford University, Stanford, California 94309 \\ ${ }^{22}$ Soongsil University, Seoul, Korea 156-743 \\ ${ }^{23}$ University of Tennessee, Knoxville, Tennessee 37996 \\ ${ }^{24}$ Tohoku University, Sendai 980, Japan \\ ${ }^{25}$ University of California at Santa Barbara, Santa Barbara, California 93106 \\ ${ }^{26}$ University of California at Santa Cruz, Santa Cruz, California 95064 \\ ${ }^{27}$ Vanderbilt University, Nashville, Tennessee 37235 \\ ${ }^{28}$ University of Washington, Seattle, Washington 98105 \\ ${ }^{29}$ University of Wisconsin, Madison, Wisconsin 53706 \\ ${ }^{30}$ Yale University, New Haven, Connecticut 06511
}

(Received 6 October 2000)

We present final measurements of the $Z$ boson-lepton coupling asymmetry parameters $A_{e}, A_{\mu}$, and $A_{\tau}$ with the complete sample of polarized $Z$ bosons collected by the SLD detector at the SLAC Linear Collider. From the left-right production and decay polar angle asymmetries in leptonic $Z$ decays we 
measure $A_{e}=0.1544 \pm 0.0060, A_{\mu}=0.142 \pm 0.015$, and $A_{\tau}=0.136 \pm 0.015$. Combined with our left-right asymmetry measured from hadronic decays, we find $A_{e}=0.1516 \pm 0.0021$. Assuming lepton universality, we obtain a combined effective weak mixing angle of $\sin ^{2} \theta_{W}^{\text {eff }}=0.23098 \pm 0.00026$.

DOI: 10.1103/PhysRevLett.86.1162

The extent of parity violation in the electroweak interaction can be probed directly in the production and decay of polarized $Z$ bosons generated by $e^{+} e^{-}$annihilation. Parity violation in $Z$ production $\left(e^{+} e^{-} \rightarrow Z\right)$ and decay into charge lepton pairs $\left(Z \rightarrow e^{+} e^{-}, \mu^{+} \mu^{-}, \tau^{+} \tau^{-}\right)$ are characterized by the $Z$ boson-lepton coupling asymmetry parameters $A_{e}, A_{\mu}$, and $A_{\tau}$. The asymmetry parameter is defined as $A_{l}=2 v_{l} a_{l} /\left(v_{l}^{2}+a_{l}^{2}\right)$, where $v_{l}$ and $a_{l}$ are the effective vector and axial-vector couplings of the $Z$ boson to the lepton (flavor "l") current, respectively. The standard model (SM) assumes lepton universality, so that all three species of leptonic asymmetry parameters are expected to be identical and directly related to the effective electroweak mixing angle $\left(\sin ^{2} \theta_{W}^{\text {eff }}\right)$, $A_{l}=2\left(1-4 \sin ^{2} \theta_{W}^{\text {eff }}\right) /\left[1+\left(1-4 \sin ^{2} \theta_{W}^{\text {eff }}\right)^{2}\right]$. The effective electroweak mixing angle depends on virtual electroweak radiative corrections including those which involve the Higgs boson and those arising from new phenomena outside of the scope of the SM. Presently, the most stringent upper bounds on the SM Higgs mass are provided by measurements of $\sin ^{2} \theta_{W}^{\mathrm{eff}}$.

The SLAC Linear Collider (SLC) produces polarized $Z$ bosons in $e^{+} e^{-}$collisions at the $Z$ resonance using a longitudinally polarized electron beam. Electron polarization $\left(P_{e}\right)$ allows us to form the left-right crosssection asymmetry to extract the initial state asymmetry parameter $A_{e}$ [1] and also enables us to directly measure the final state asymmetry parameter $A_{e}$ [1] and also enables us to directly measure the final state asymmetry parameter $A_{l}$ for lepton $l$ using the left-right forward-backward asymmetry [2] $\left(\tilde{A}_{F B}=\frac{3}{4}\left|P_{e}\right| A_{l}\right)$. Experiments at the $Z$ resonance without beam polarization [3] have measured the product of initial and final state asymmetry parameters $\left(A_{F B}=\frac{3}{4} A_{e} \cdot A_{l}\right)$. Those same experiments have also measured the tau polarization [3] which yields $A_{e}$ and $A_{\tau}$ separately. The SLC beam polarization enables us to present the only direct measurement of $A_{\mu}$. With $75 \%$ beam polarization, the left-right forward-backward asymmetries yield a statistical precision equivalent to measurements using a 25 times larger event sample with the unpolarized forward-backward asymmetry.

In this Letter, we report new results on direct measurements of the asymmetry parameters $A_{e}, A_{\mu}$, and $A_{\tau}$ using leptonic $Z$ decays. The measurements are based on the $3.8 \times 10^{5} \mathrm{Zs}$ collected during $1996-1998$ by the SLAC Large Detector (SLD) experiment at the SLC. These results are combined with earlier leptonic asymmetry measurements [2] (based on $1.5 \times 10^{5} \mathrm{Zs}$ ) and the more precise left-right asymmetry measurement using $Z$ decays
PACS numbers: 13.38.Dg, 12.15.Ji, 13.10.+q, 13.88.+e

to hadrons [1], to give final measurements based on the complete sample of polarized $Z$ bosons.

This analysis relies on the Compton polarimeter [1,4], tracking by the vertex detector and the central drift chamber [5], and the liquid argon calorimeter (LAC) [6]. Details about the SLC, the polarized electron source, and SLC operation with a polarized beam can be found in Ref. [7]. Only the details most relevant to this analysis are mentioned here.

In our previous measurements [2], the analysis was restricted to the polar-angle range of $|\cos \theta|<0.7$ due to decreasing tracking and trigger efficiency for muon-pair final states beyond this region, even though the high $|\cos \theta|$ region is very sensitive to the asymmetry parameters. In 1996 we installed an upgraded vertex detector (VXD3) [8] and a new trigger system for forward muon-pair events. The improved acceptance of VXD3 allows highly efficient track finding up to $|\cos \theta|=0.9$ [9]. The new trigger for $\mu^{+} \mu^{-}$events covers the angular range up to $|\cos \theta|<$ 0.95 by requiring two back-to-back tracks that pass through the interaction point and reach the end cap Warm Iron Calorimeter [10].

Polarization-dependent lepton asymmetries are easily computed from $e_{L, R}^{-}+e^{+} \rightarrow Z^{0} \rightarrow l^{-}+l^{+}$, where $l$ represents an electron, a muon, or a tau lepton. The differential cross section is expressed as follows:

$$
\begin{aligned}
\frac{d}{d x} \sigma_{Z}\left(x, s, P_{e} ; A_{e}, A_{l}\right) \equiv f_{Z}(s) \Omega_{Z}\left(x, P_{e} ; A_{e}, A_{l}\right) \\
=f_{Z}(s)\left[\left(1-P_{e} A_{e}\right)\left(1+x^{2}\right)\right. \\
\left.+\left(A_{e}-P_{e}\right) A_{l} 2 x\right],
\end{aligned}
$$

where $s$ is the squared center-of-mass energy and $x=$ $\cos \theta$ gives the direction of the outgoing lepton $\left(l^{-}\right)$with respect to the electron-beam direction. (For $P_{e}$, we use the convention that left-handed bunches have negative sign.) Photon exchange terms and, if the final state leptons are electrons, $t$-channel contributions have to be taken into account. The leptonic asymmetry parameters which refer to the initial and final state lepton appear in this expression as $A_{e}$ and $A_{l}$, respectively. It was determined that $\left|P_{e}\right|=76.16 \pm 0.40 \%$ and $72.92 \pm 0.38 \%$ for the 1996 and 1997-1998 runs, respectively [1].

Leptonic $Z$ decay candidates are required to have between two and eight charged tracks, each of which must pass within $1 \mathrm{~cm}$ of the nominal $e^{+} e^{-}$interaction point. This excludes most hadronic $Z$ decays, which have an average charged multiplicity of approximately 20 . One hemisphere must have a net charge 1 and the other a net charge 
-1 to ensure unambiguous assignment of the scattering angle. Each event is assigned a polar-production angle with respect to the electron beam direction based on the thrust axis $\left(\cos \theta_{\text {thrust }}\right)$ defined by the charged tracks and we require $\left|\cos \theta_{\text {thrust }}\right|<0.9(0.8)$ for $1997-1998$ (1996) data.

A single additional cut is required to select the $e^{+} e^{-}$ final state. We consider the highest momentum track in each hemisphere and require the sum of the associated energies deposited in the LAC to exceed $65 \mathrm{GeV}$. The $e^{+} e^{-}$candidates have a small contamination $(0.7 \%)$ from $\tau^{+} \tau^{-}$events.

For events of the type $Z \rightarrow \mu^{+} \mu^{-}$, we require the invariant mass of the charged tracks (assumed pion mass) to be greater than $70 \mathrm{GeV} / c^{2}$. This removes most $Z \rightarrow$ $\tau^{+} \tau^{-}$events and virtually all two-photon and hadronic $Z$ decay events. We remove the $e^{+} e^{-}$final state by requiring the energy deposited in the LAC by the highest momentum track in each hemisphere to be less than $14 \mathrm{GeV}$. The muon-pair sample has a very small contamination $(0.2 \%)$ from $\tau^{+} \tau^{-}$final states.

The tau-pair final state selection requires the event mass to be less than $70 \mathrm{GeV} / c^{2}$ to remove $\mu^{+} \mu^{-}$final states. The maximum energy per hemisphere in the LAC associated with a charged track is required to be less than

$39 \mathrm{GeV}(33 \mathrm{GeV})$ for $\cos \theta<0.7(>0.7)$ to reject $e^{+} e^{-}$ final states. Two-photon events are suppressed by requiring the angle between the total track momenta of the two hemispheres to be greater than $160^{\circ}$ and by requiring one charged track to have momentum greater than $4 \mathrm{GeV} / c$. The remaining background from hadronic $Z$ decays is suppressed by requiring each hemisphere invariant mass, measured using charged tracks, to be less than $1.6 \mathrm{GeV} / c^{2}$. The tau-pair candidates have some contamination from muon pair (2.9\%), electron pair $(0.9 \%)$, two-photon events $(0.9 \%)$, and hadronic final states $(0.6 \%)$.

Table I summarizes the selection efficiencies, backgrounds, and numbers of selected candidates for $e^{+} e^{-}$, $\mu^{+} \mu^{-}$, and $\tau^{+} \tau^{-}$final states. Figure 1 shows the $\cos \theta$ distributions for $e^{+} e^{-}, \mu^{+} \mu^{-}$, and $\tau^{+} \tau^{-}$candidates for the 1997-1998 data. The asymmetries in the 1996 data are similar but have smaller acceptance $(|\cos \theta| \leq 0.8)$.

We perform a maximum likelihood fit, event by event, to incorporate the contributions of all the terms in the cross section and to include the effect of initial state radiation. We define three likelihood functions for individual lepton final states. $A_{e}$ and $A_{\mu}\left(A_{\tau}\right)$ are derived from $\mu^{+} \mu^{-}$ $\left(\tau^{+} \tau^{-}\right)$final states. These $A_{e}$ results are combined with the number obtained from $e^{+} e^{-}$final states.

The likelihood function for muon- and tau-pair final states is defined as follows:

$$
\mathcal{L}\left(x, s, P_{e} ; A_{e}, A_{l}\right)=\int d s^{\prime} H\left(s, s^{\prime}\right)\left\{\frac{d}{d x} \sigma_{Z}\left(x, s^{\prime}, P_{e} ; A_{e}, A_{l}\right)+\frac{d}{d x} \sigma_{Z \gamma}\left(x, s^{\prime}, P_{e} ; A_{e}, A_{l}\right)+\frac{d}{d x} \sigma_{\gamma}\left(x, s^{\prime}\right)\right\},
$$

where $A_{e}$ and $A_{l}\left(=A_{\mu}\right.$ or $\left.A_{\tau}\right)$ are free parameters and $H\left(s, s^{\prime}\right)$ is a radiator function. The integration over $s^{\prime}$ is done with the program MIZA [11] to take into account the initial state radiation. The spread in the beam energy has a negligible effect. $\left(d \sigma_{Z} / d x\right)(\ldots),\left(d \sigma_{\gamma} / d x\right)(\ldots)$, and $\left(d \sigma_{Z \gamma} / d x\right)(\ldots)$ are the tree-level differential cross sections for $Z$ exchange, photon exchange, and their interference. The integration is performed before the fit to obtain the coefficients $\bar{f}_{Z}, \bar{f}_{Z \gamma}$, and $\bar{f}_{\gamma}$, and the likelihood function becomes

$$
\begin{aligned}
\mathcal{L}\left(x, s, P_{e} ; A_{e}, A_{t}\right)= & \bar{f}_{Z}(s) \Omega_{Z}\left(x, P_{e} ; A_{e}, A_{l}\right) \\
& +\bar{f}_{Z \gamma}(s) \Omega_{Z \gamma}\left(x, P_{e} ; A_{e}, A_{l}\right) \\
& +\bar{f}_{\gamma}(s) \Omega_{\gamma}(x) .
\end{aligned}
$$

These coefficients give the relative sizes of the three terms at the SLC center-of-mass energy $(\sqrt{s}=91.237 \pm$ $0.029 \mathrm{GeV}$ for the 1997-1998 run and $91.26 \pm 0.03 \mathrm{GeV}$ for 1996) [1].

The $e^{+} e^{-}$final state includes both $s$-channel and $t$-channel $Z$ and photon exchanges which yields four amplitudes and ten cross-section terms. All ten terms are energy dependent. We define a maximum likelihood function for $e^{+} e^{-}$final states by modifying Eqs. (1) and (2) to include all ten terms. The integration over $s^{\prime}$ is performed with DMIBA [12] to obtain the coefficients for the relative size of the ten terms.
There are several systematic effects which can bias the results. The uncertainties associated with these effects are summarized in Table II and are small compared to the statistical uncertainties. The uncertainty on the beam polarization is correlated among all the measurements and corresponds to an uncertainty on $A_{l}$ of \pm 0.0008 . The uncertainty in the amount of background and its effect on the fitted parameters are taken into account. The background contaminations have been derived from detailed Monte Carlo simulations as well as from studying the effect of cuts in background-rich samples of real data. The radiative correction and their systematic errors are estimated using the MIZA [11] and DMIBA [12] programs whose inherent 1 per mil precision leads to negligible effects compared to our $\sqrt{s}$ uncertainty. The uncertainty in the asymmetry parameters due to a $\pm 1 \sigma$ variation of $\sqrt{s}$ (which affects radiative corrections) is of the order of $10^{-4}$, except for the $A_{e}$ determination from $e^{+} e^{-}$final states for which it is of the order of $10^{-3}$.

The dominant systematic error in the tau analysis results from the V-A structure of tau decay [13], which introduces a selection bias in our analysis. For example, if both taus decay to $\pi \nu$, helicity conservation requires that both pions generally have lower momentum for a left-handed $\tau^{-}$and right-handed $\tau^{+}$and higher momentum otherwise. This effect, which biases the reconstructed 
TABLE I. Summary of event selections, efficiency, and purity for $e^{+} e^{-} \rightarrow l^{+} l^{-}$for the 1997-1998 (1996) data.

\begin{tabular}{cccc}
\hline \hline $\begin{array}{c}\text { Event } \\
\text { sample }\end{array}$ & $\begin{array}{c}\text { Background as } \% \\
\text { of selected events }\end{array}$ & $\begin{array}{c}\text { Efficiency in } \\
|\cos \theta|<0.9(|\cos \theta|<0.8)\end{array}$ & $\begin{array}{c}\text { No. of selected } \\
\text { events }\end{array}$ \\
\hline$e^{+} e^{-} \rightarrow e^{+} e^{-}$ & $0.7 \%(0.8 \%) \tau^{+} \tau^{-}$ & $75 \%(87 \%)$ & $15675(2052)$ \\
$Z \rightarrow \mu^{+} \mu^{-}$ & $0.2 \%(0.2 \%) \tau^{+} \tau^{-}$ & $77 \%(83 \%)$ & $11431(1625)$ \\
$Z \rightarrow \tau^{+} \tau^{-}$ & $0.9 \%(0.7 \%) e^{+} e^{-}$ & & $10841(1494)$ \\
& $2.9 \%(2.2 \%) \mu^{+} \mu^{-}$ & $70 \%(77 \%)$ & \\
& $0.9 \%(0.9 \%)$ two photon & & \\
& $0.6 \%(0.3 \%)$ hadrons & & \\
\hline \hline
\end{tabular}

event mass, is large at the SLD because the high beam polarization induces a very high and asymmetric tau polarization as a function of polar angle. Using detailed Monte Carlo simulation [14,15], we find an overall shift in $A_{\tau}$ of $+0.0182 \pm 0.0018(+0.0183 \pm 0.0017)$ for the 1997-1998 (1996) runs due to the effect of the V-A structure, where the uncertainty is from Monte Carlo statistics. The value extracted from the fit must be reduced by this amount. The value of $A_{e}$ extracted from $\tau^{+} \tau^{-}$final states is not affected since the overall relative efficiencies for left-handed beam and right-handed beam events are not

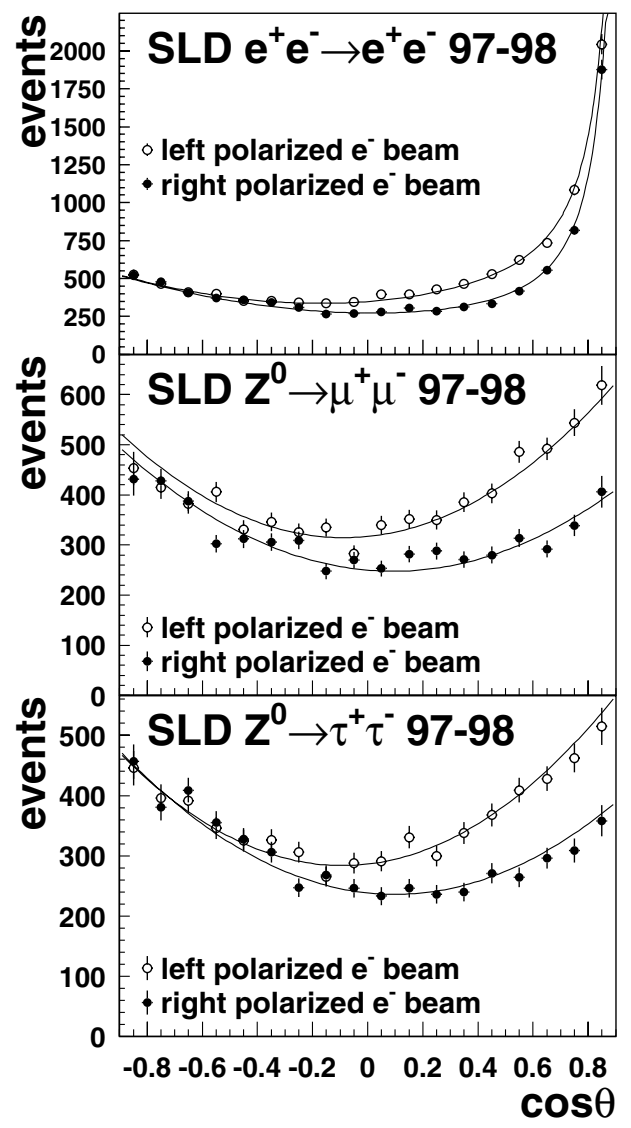

FIG. 1. Polar-angle distributions for $Z$ decays to $e, \mu$, and $\tau$ pairs for the 1997-1998 SLD run. The solid line represents the fit, while the points with error bars show the data in bins of 0.1 in $\cos \theta_{\text {thrust }}$. For $\left|\cos \theta_{\text {thrust }}\right|>0.7$, the data are corrected for a decrease in the detection efficiency with increasing $\left|\cos \theta_{\text {thrust }}\right|$. changed significantly (only the polar-angle dependence of the efficiencies is changed).

Tracks are less well measured at very high $|\cos \theta|$ and charge confusion for these tracks dilutes the asymmetries. We estimate this effect by comparing the numbers of opposite sign back-to-back tracks with same-sign pairs. The uncertainty is found to be \pm 0.0007 and \pm 0.0011 for $A_{\mu}$ and $A_{\tau}$, respectively. A small detector-induced forwardbackward asymmetry would also introduce a small bias for $A_{\tau}$. Using a two-photon enriched data sample, we find a small forward-backward asymmetry effect in the momentum distribution of negatively signed charged tracks $(\sim 1.0 \mathrm{GeV} / c)$. We estimate this causes a systematic uncertainty of \pm 0.0004 for $A_{\tau}$, while the effect is negligible for $A_{e}$ and $A_{\mu}$. The selection efficiency as a function of polar angle is another possible source of bias in $A_{l}$. If this efficiency is symmetric about $\cos \theta=0$ then $A_{l}$ is unaffected for muons and taus. However, the maximum likelihood fit for the $e^{+} e^{-}$final state may be affected even for a symmetric efficiency, if it is not uniform. This systematic uncertainty is estimated to be \pm 0.0002 for $A_{e}$ by using the Monte Carlo simulation to compare the nominal result with the result for $100 \%$ selection efficiency for the $e^{+} e^{-}$final state. We have also studied the effect of the uncertainty in the thrust axis determination, which also includes the uncertainty from the final state radiation, and found that the contribution is negligible.

We find the results for $A_{e}, A_{\mu}$, and $A_{\tau}$ using the 19961998 SLD runs to be $A_{e}=0.1549 \pm 0.0066$ (stat) \pm 0.0013 (syst),$\quad A_{\mu}=0.152 \pm 0.016$ (stat) \pm 0.001 (syst), and $A_{\tau}=0.121 \pm 0.017$ (stat) \pm 0.003 (syst), respectively. We combine these results with our previous leptonic asymmetry measurements [2], accounting for small effects due to correlations in systematic uncertainties (polarization and average SLD center-of-mass energy). From purely leptonic final states, we obtain $A_{e}=0.1544 \pm$ 0.0060 . We also combine the $A_{e}$ result with the left-right asymmetry measurement using $Z$ decays to hadrons $\left(A_{L R}^{0} \equiv A_{e}\right)[1]$ and obtain

$$
\begin{aligned}
A_{e} & =0.1516 \pm 0.0021\left(\text { with } A_{L R}^{0}\right), \\
A_{\mu} & =0.142 \pm 0.015 \\
A_{\tau} & =0.136 \pm 0.015
\end{aligned}
$$


TABLE II. Summary of statistical and systematic uncertainties in units of $10^{-4}$ for the 1997-1998 (1996) data.

\begin{tabular}{cccccc}
\hline \hline Source & $A_{e}^{e}$ & $A_{e}^{\mu}$ & $A_{e}^{\tau}$ & $A_{\mu}^{\mu}$ & $A_{\tau}^{\tau}$ \\
\hline Statistics & $110(280)$ & $130(330)$ & $130(340)$ & $180(470)$ & $180(480)$ \\
Polarization & $8(8)$ & $8(8)$ & $8(8)$ & $8(8)$ & $8(8)$ \\
Backgrounds & $5(3)$ & $\ldots$ & $13(14)$ & $\ldots$ & $14(13)$ \\
Radiative correction & $23(17)$ & $2(2)$ & $2(2)$ & $3(1)$ & $2(2)$ \\
V-A & $\ldots$ & $\ldots$ & $\ldots$ & $\ldots$ & $18(17)$ \\
Charge confusion & $\ldots$ & $\ldots$ & $\ldots$ & $7(-)$ & $11(1)$ \\
Detector asymmetry & $\ldots$ & $\ldots$ & $\ldots$ & $\ldots$ & $4(4)$ \\
Nonuniform efficiency & $2(-)$ & $\cdots$ & $\cdots$ & $\cdots$ & $\cdots$ \\
\hline \hline
\end{tabular}

Our results are consistent with lepton universality. Assuming universality, we combine these results into $A_{l}$, which in the context of the standard model is simply related to the electroweak mixing angle:

$$
\begin{gathered}
A_{l}=0.15130 \pm 0.00207, \\
\sin ^{2} \theta_{W}^{\text {eff }}=0.23098 \pm 0.00026 .
\end{gathered}
$$

Within the context of the SM, the above result can be used to constrain the mass of the Higgs boson. We use the measured $Z$ boson [3] and top quark [16] masses, a determination of $\alpha\left(M_{Z}^{2}\right)$ [17], and the ZFITTER 6.23 program [18] to obtain a $95 \%$ confidence level upper bound of $147 \mathrm{GeV} / c^{2}$.

In conclusion, we have presented direct measurements of the $Z$ boson-lepton coupling asymmetries $A_{e}, A_{\mu}$, and $A_{\tau}$ using $e^{+} e^{-} \rightarrow e^{+} e^{-}, \mu^{+} \mu^{-}, \tau^{+} \tau^{-}$events produced with a longitudinally polarized electron beam during the 1996-1998 SLD runs. These results are combined with our previously published results, yielding SLD's final result for the weak mixing angle. This is presently the most precise available determination of this quantity.

We thank the personnel of the SLAC accelerator department and the technical staffs of our collaborating institutions for their outstanding efforts on our behalf. This work was supported by the Department of Energy, the National Science Foundation, the Istituto Nazionale di Fisica Nucleare of Italy, the Japan-U.S. Cooperative Research Project on High Energy Physics, and the Science and Engineering Research Council of the United Kingdom.

[1] SLD Collaboration, K. Abe et al., Phys. Rev. Lett. 84, 5945 (2000); SLD Collaboration, K. Abe et al., Phys. Rev. Lett. 78, 2075 (1997); SLD Collaboration, K. Abe et al., Phys.
Rev. Lett. 73, 25 (1994); SLD Collaboration, K. Abe et al., Phys. Rev. Lett. 70, 2515 (1993).

[2] SLD Collaboration, K. Abe et al., Phys. Rev. Lett. 79, 804 (1997).

[3] The LEP Collaboration, the LEP Electroweak Working Group, The SLD Heavy Flavour and Electroweak Working Groups, Report No. CERN-EP/2000-016, 2000.

[4] R. King, SLAC-Report-452, 1994; A. Lath, SLACReport-454, 1994; E. Torrence, SLAC-Report-509, 1997; D. Onoprienko, SLAC-Report-556, 2000.

[5] M. J. Fero et al., Nucl. Instrum. Methods Phys. Res., Sect. A 367, 111 (1995).

[6] D. Axen et al., Nucl. Instrum. Methods Phys. Res., Sect. A 328, 472 (1993).

[7] M. Woods, in Proceedings of the 12th International Symposium on High Energy Spin Physics (SPIN96), The Netherlands, 1996, edited by C. W. Jager et al. (World Scientific, Singapore, 1997), p. 621.

[8] K. Abe et al., Nucl. Instrum. Methods Phys. Res., Sect. A 400, 287 (1997); SLD Collaboration, N. B. Sinev et al., IEEE Trans. Nucl. Sci. 44, 587 (1997); SLD Collaboration, J. E. Brau, Nucl. Instrum. Methods Phys. Res., Sect. A 418, 52 (1998).

[9] SLD Collaboration, T. Abe, Nucl. Instrum. Methods Phys. Res., Sect. A 447, 90 (2000).

[10] A. C. Benvenuti et al., Nucl. Instrum. Methods Phys. Res., Sect. A 276, 94 (1989).

[11] M. Martinez et al., Z. Phys. C 49, 645 (1991).

[12] M. Martinez and R. Miquel, Z. Phys. C 53, 115 (1992).

[13] Y. Tsai, Phys. Rev. D 4, 2821 (1971).

[14] S. Jadach, B. F. Ward, and Z. Was, Comput. Phys. Commun. 79, 503 (1994).

[15] S. Jadach et al., Comput. Phys. Commun. 76, 361 (1993).

[16] R. Partridge, in Proceedings of the XXIXth International Conference on High Energy Physics, Vancouver, Canada, 1998 (World Scientific, Singapore, 1999), p. 107.

[17] A.D. Martin, J. Outhwaite, and M.G. Ryskin, hep-ph/ 0008078. We have chosen this value because it provides the least stringent limit of all the presently available evaluations of $\alpha\left(M_{Z}^{2}\right)$.

[18] D. Bardin et al., Report No. DESY 99-070, 1999. 